\title{
Regulation of embryonic stem cell fate by heparan sulfate proteoglycans
}

\author{
Victor Nurcombe ${ }^{1}$, Torben Helledie ${ }^{1}$, Simon M Cool $^{1}$ \\ ${ }^{1}$ Stem Cell and Tissue Repair Group, Institute of Molecular and Cell Biology, Biopolis, Singapore 138673
}

Pluripotent embryonic stem cells (ESC) are valuable tools for understanding developmental processes and have tremendous clinical potential if their lineage commitment can be tightly controlled. Use of exogenous growth factors is currently employed as a means to control ESC growth and diffentiation that have also been shown to affect pluripotency. The activity of these growth factors is regulated by heparan sulfate proteoglycans (HSPGs) that are present on the cell surface or in the extracellular matrix (ECM). The HSPGs consist of a defining core protein to which linear glycosaminoglycan side chains of heparan sulfate (HS) sugars are covalently attached. Importantly HS bioactivity and growth factor affinity depends of its pattern of sulfation that is known to change during tissue development and maturation. Thus, an understanding of HS sulfation patterns during ESC culturing will be an invaluable tool in controlling ESC proliferation and differentiation. In this study we examined the expression of enzymes responsible for HS biosynthesis and sulfation during ESC growth and differentiation using qPCR. In addition, we performed a biochemical analysis of HS chains produced during ESC culture. Furthermore, we used chemical and enzymatic treatments to alter the expression of HSPGs and have characterized the effect on ESC proliferation and differentiation. We show that HSPGs are vital in regulating ESC fate and provide a novel tool for controlling growth factor mediated effects on in vitro cultures of ESCs.

Cell Research (2008) 18:s53. doi: 10.1038/cr.2008.143; published online 4 August 2008

Correspondence: Torben Helledie

E-mail: thelledie@imcb.a-star.edu.sg 